\author{
Mohamed Khalifa
}

\title{
Note on valuative dimension in power series rings
}

Received: 3 January 2014 / Accepted: 2 July 2014 / Published online: 9 August 2014

(C) The Author(s) 2014. This article is published with open access at Springerlink.com

\begin{abstract}
We give necessary and sufficient conditions for the power series ring $R\left[\left[x_{1}, \ldots, x_{n}\right]\right]$ to be a Jaffard domain, where $R$ is an almost pseudo-valuation domain.
\end{abstract}

Mathematics Subject Classification 13F25 - 13C15 - 13F05 $\cdot 13 \mathrm{~A} 15$

$$
\text { المعطي شروطا ضرورية وكافية على مجال شبه تقييمي تقريباً R بحيث تكون حلقة منسلسلات القوى [] }
$$

All rings considered below are (commutative with identity) integral domains. The dimension of a ring $R$, denoted by $\operatorname{dim} R$, means its Krull dimension. In [10], Jaffard defines the valuative dimension, denoted by $\operatorname{dim}_{v} R$, of an integral domain $R$ to be the maximal rank of the valuation overrings of $R$. Following [1], an integral domain $R$ is said to be a Jaffard ring if $\operatorname{dim}_{v} R=\operatorname{dim} R<\infty$. Most standard examples of Jaffard domains are finite-dimensional Noetherian domains and finite-dimensional Prüfer domains [10].

In this paper, we are interested in when the power series ring $R[[x]]$ is a Jaffard domain. It was shown by Arnold in [2] that if a ring $R$ fails to have the SFT(strong finite type)-property, then $R[[x]]$ has infinite dimension, and so has infinite valuative dimension. Recall that an ideal $I$ of a ring $R$ is an SFT-ideal if there exist a positive integer $k$ and a finitely generated ideal $F$ of $R$ such that $F \subseteq I$ and $x^{k} \in F$ for each $x \in I$; moreover, if each ideal of $R$ is an SFT-ideal, then we say that $R$ is an SFT-ring.

Let $R$ be an integral domain. When is $R[[x]]$ a Jaffard domain? The answer is known in two cases. First, if $R$ is a Noetherian ring with finite dimension, then $R[[x]]$ is also Noetherian with finite dimension [7, Lemma 2.6], and so a Jaffard domain. The second case was shown by Kang and Park. In [11], they computed the dimension of mixed extension $\left.\left.R\left[x_{1}\right]\right] \ldots\left[x_{n}\right]\right]$ where $R$ is a finite dimensional SFT-Prüfer domain. In [15], Park generalized the result to the case, where $R$ is a finite dimensional SFT-globalized pseudo-valuation domain (for short, GPVD) as shown in the following:

Theorem 1 [15, Theorem 2.15] Let $R$ be an m-dimensional SFT-GPVD with associated Prüfer domain $T$ and let $I=(R: T)$. For each maximal ideal $M$ of $R$, let $N_{M}$ be the maximal ideal of $T$ such that $N_{M} \cap R=M$, set $k_{M}:=R / M$ and $K_{M}:=T / N_{M}$, and let $k_{0, M}$ denote the maximal separable extension of $k_{M}$ in $K_{M}$. Assume that $\left.\left[x_{i}\right]\right]=\left[\left[x_{i}\right]\right]$ for some $i$. Then, $\left.\left.\operatorname{dim} R\left[x_{1}\right]\right] \ldots\left[x_{n}\right]\right]=n m+1$ if for each maximal ideal $M$ of $R$ with $h t M=m$ and $M \supseteq I, K_{M}$ has finite exponent over $k_{0, M}$, and $\left[k_{0, M}: k_{M}\right]<\infty$ and $\left.\left.\operatorname{dim} R\left[x_{1}\right]\right] \ldots\left[x_{n}\right]\right]=n m+n$ otherwise.

M. Khalifa ( $\varangle)$

Department of Mathematics, Ecole Supérieure des Sciences et de Technologie,

Hammam Sousse 4011, Tunisia

E-mail: kmhoalg@yahoo.fr 
Recall that an integral domain $R$ is a GPVD [6] if there exists a Prüfer overring $T$ of $R$ such that (i) $R \subseteq T$ is a unibranched extension, and (ii) there exists a nonzero radical ideal $I$ common to $T$ and $R$ such that the rings $T / I$ and $R / I$ are zero-dimensional. The domain $T$ is uniquely determined [6, page 156] and is called the associated Prüfer domain of $R$. Most standard examples of GPVD are valuation domains, PVDs and Prüfer domains [6]. It is well known that $\operatorname{dim}_{v} R=$ the limit of the sequence $(\operatorname{dim} R[n]-n)$ [5] where $R[n]$ means the ring of polynomials in $n$ indeterminates over $R$. It follows immediately from Park's result that:

Corollary 2 Let $R$ be a GPVD (which is not a field) with associated Prïfer domain $T$ and let $I=(R: T)$. For each maximal ideal $M$ of $R$, let $N_{M}$ be the maximal ideal of $T$ such that $N_{M} \cap R=M$; set $k_{M}:=R / M$ and $K_{M}:=T / N_{M}$, and let $k_{0, M}$ denote the maximal separable extension of $k_{M}$ in $K_{M}$. For $m \geq 1$, the following assertions are equivalent:

(1) $R\left[\left[x_{1}, \ldots, x_{m}\right]\right]$ is a Jaffard domain.

(2) $\operatorname{dim}_{v} R\left[\left[x_{1}, \ldots, x_{m}\right]\right]<\infty$.

(3) $R$ is a one-dimensional SFT-ring, and for each maximal ideal $M$ of $R$ with $M \supseteq I, K_{M}$ has finite exponent over $k_{0, M}$ and $\left[k_{0, M}: k_{M}\right]<\infty$.

In this paper, we are interested in the case where $R$ is an almost pseudo-valuation domain (for short, APVD). Recall that an integral domain $R$ is called an almost pseudo-valuation domain if $R$ is quasi-local with maximal ideal $M$ such that $(M: M)$ is a valuation overring of $R$ with $M$ primary to the maximal ideal of $(M: M)$ [3]. A domain $R$ is called $*$-domain if it has (at least) a height one prime ideal and for each height one prime ideal $P$ of $R, R$ satisfies the ascending chain condition on ideals of the form $a P$ (where $a \in R$ ) [12]. We proved that for an APVD $R$ with nonzero finite dimension, $R\left[\left[x_{1}, \ldots, x_{n}\right]\right]$ has finite dimension if and only if $R$ is a residually $*$-domain (i.e., $R / P$ is a $*$-domain for each nonmaximal prime ideal $P$ of $R$ ) [12, Theorem 2.9], if and only if the integral closure of $R$ is an SFT-ring [13, Theorem 2.3]. Note that every PVD is an APVD, APVD's class contains strictly the PVD's class [3, Example 3.9] and an APVD is a GPVD if and only if it is a PVD [13, beginning of Section 3]. The following result gives us a necessary and sufficient condition on an APVD $R$ so that $R[[x]]$ is a Jaffard domain.

Theorem 3 Let $R$ be an APVD with maximal ideal $M$, residue field $K$ and nonzero dimension, $L$ be the residue field of $(M: M)$ and $K_{0}$ be the maximal separable extension of $K$ in $L$. For $m \geq 1$, the following assertions are equivalent:

(1) $R\left[\left[x_{1}, \ldots, x_{m}\right]\right]$ is a Jaffard domain.

(2) $\operatorname{dim}_{v} R\left[\left[x_{1}, \ldots, x_{m}\right]\right]<\infty$.

(3) $R$ is one-dimensional with SFT-integral closure, $\left[K_{0}: K\right]<\infty$, and $L$ has finite exponent over $K_{0}$.

(4) $R$ satisfies the ascending chain condition on principal ideals, the integral closure of $R$ is an SFT-ring, $\left[K_{0}: K\right]<\infty$, and $L$ has finite exponent over $K_{0}$.

Proof Let $N$ be the maximal ideal of the valuation domain $V:=(M: M)$.

(1) $\Rightarrow$ (2) trivial. (2) $\Rightarrow$ (3) Since $\operatorname{dim} R\left[\left[x_{1}, \ldots, x_{m}\right]\right]<\infty, R$ has an SFT-integral closure and $N^{s} \subseteq M$ for some positive integer $s$ by Khalifa and Benhissi [13, Theorem 2.3]. Thus, the prime ideal $N\left[\left[x_{1}, \ldots, x_{m}\right]\right]$ is minimal over $M\left[\left[x_{1}, \ldots, x_{m}\right]\right]$ in $V\left[\left[x_{1}, \ldots, x_{m}\right]\right]$. Then $\operatorname{dim} R\left[\left[x_{1}, \ldots, x_{m}\right]\right][n] \geq$ $h t_{V\left[\left[x_{1}, \ldots, x_{m}\right]\right][1]} N\left[\left[x_{1}, \ldots, x_{m}\right]\right][1]+\inf \{d, n-1\}+\operatorname{dim} K\left[\left[x_{1}, \ldots, x_{m}\right]\right][n]=(m+1)(\operatorname{dim} R-1)+$ $1+\inf \{d, n-1\}+m+n$ by Cahen [5, Lemme 3], Khalifa and Benhissi [13, Lemma 2.1], and Park [15, Lemma 2.7], where $d$ is the transcendence degree of $L\left[\left[x_{1}, \ldots, x_{m}\right]\right]$ over $K\left[\left[x_{1}, \ldots, x_{m}\right]\right]$. If $d=\infty$, then $\operatorname{dim} R\left[\left[x_{1}, \ldots, x_{m}\right]\right][n] \geq(m+1)(\operatorname{dim} R-1)+1+n-1+m+n \geq 2 n$ which contradicts that $R\left[\left[x_{1}, \ldots, x_{m}\right]\right]$ has finite valuative dimension. Thus, $d<\infty$ and so $\left[K_{0}: K\right]<\infty$ and $L$ has finite exponent over $K_{0}$ by Park [14, Lemma 2.1]. To show that $R$ is 1-dimensional, we apply again [5, Lemme 3], [13, Lemma 2.1], and [15, Lemma 2.7]. Then we have $\operatorname{dim} R\left[\left[x_{1}, \ldots, x_{m}\right]\right][n]-n \geq h t_{V\left[\left[x_{1}, \ldots, x_{m}\right]\right][n]} N\left[\left[x_{1}, \ldots, x_{m}\right]\right][n]+$ $\operatorname{dim} K\left[\left[x_{1}, \ldots, x_{m}\right]\right][n]-n=(n+m)(\operatorname{dim} R-1)+1+m+n-n=n(\operatorname{dim} R-1)+m \operatorname{dim} R+1$. Hence, $\operatorname{dim} R=1$. (3) $\Rightarrow$ (4) see [13, Lemma 2.2 and Theorem 2.3]. (4) $\Rightarrow$ (1) Recall that a domain $D$ is called Archimedean in case $\bigcap_{n=1}^{\infty} a^{n} D=0$ for each nonunit $a$ of $D$. It is well known that rings satisfying the ascending chain condition on principal ideals are Archimedean and a divided Archimedean domain must have Krull dimension $\leq 1$, and so $\operatorname{dim} R=1$ by Badawi and Houston [3, Proposition 3.2]. Thus, $N^{s} \subseteq M$ for some positive integer $s$ by Khalifa and Benhissi [13, Lemma 2.2]. By Park [14, Lemma 2.1], $L\left[\left[x_{1}, \ldots, x_{m}\right]\right][n]$ is integral over $K\left[\left[x_{1}, \ldots, x_{m}\right]\right][n]$. Thus, $V\left[\left[x_{1}, \ldots, x_{m}\right]\right][n]$ is integral over $R\left[\left[x_{1}, \ldots, x_{m}\right]\right][n]$. Hence, $\operatorname{dim} R\left[\left[x_{1}, \ldots, x_{m}\right]\right][n]-n=(n+m) \operatorname{dim} R+1-n=m+1$ for every positive integer $n$ by Kang and Park [11, Theorem 14]. Then $\operatorname{dim}_{v} R\left[\left[x_{1}, \ldots, x_{m}\right]\right]=m+1(<\infty)=\operatorname{dim} R\left[\left[x_{1}, \ldots, x_{m}\right]\right]$. 
Remark 4 Park [15] showed that if $R$ is an $m$-dimensional SFT-PVD with maximal ideal $M$ and $\left.\left[x_{i}\right]\right]=\left[\left[x_{i}\right]\right]$ for some $i$, then $\left.\left.\operatorname{dim} R\left[x_{1}\right]\right] \ldots\left[x_{n}\right]\right]=n m+1$ if $L$ has finite exponent over $K_{0}$ and $\left[K_{0}: K\right]<\infty$ (where $K=R / M, L$ is the residue field of $(M: M)$ and $K_{0}$ is the maximal separable extension of $K$ in $\left.L\right)$, and $\left.\left.\operatorname{dim} R\left[x_{1}\right]\right] \ldots\left[x_{n}\right]\right]=n m+n$ otherwise.

We show that Park's results [15, Lemma 2.8 and Theorem 2.9] remain true also if we replace a finitedimensional SFT-PVD by a nonzero finite-dimensional APVD $R$ with SFT-integral closure. The proof is the same as Park's proof (in the proof, we need also [13, Theorem 2.3]: $N^{s} \subseteq M$ for some positive integer $s>0$ where $M$ is the maximal ideal of $R$ and $N$ is the maximal ideal of the ring $(M: M)$ ), and so we do not rewrite it. Then, we have: if $R$ is an APVD with SFT-integral closure, maximal ideal $M$, nonzero finite dimension $m$, residue field $K, L$ is the residue field of $(M: M), K_{0}$ is the maximal separable extension of $K$ in $L$ and $\left.\left[x_{i}\right]\right]=\left[\left[x_{i}\right]\right]$ for some $i$, then $\left.\left.\operatorname{dim} R\left[x_{1}\right]\right] \ldots\left[x_{n}\right]\right]=n m+1$ if $L$ has finite exponent over $K_{0}$ and $\left[K_{0}: K\right]<\infty$, and $\left.\left.\operatorname{dim} R\left[x_{1}\right]\right] \ldots\left[x_{n}\right]\right]=n m+n$ otherwise. This gives us another proof for Theorem 3 using Park's technique. domain.

The following example shows that there exists a non-Noetherian domain $R$ such that $R[[x]]$ is a Jaffard

Example 5 If $R$ is a one-dimensional Prüfer domain, then $R\left[\left[x_{1}, \ldots, x_{n}\right]\right]$ is a Jaffard domain if and only if $R$ is Noetherian (because any one-dimensional SFT-Prüfer domain is a Dedekind domain). This is not the case for PVDs. More generally, there exists a non-Noetherian PVD $R$ such that $R[[x]]$ is a Jaffard domain. Let $F$ be a field with nonzero characteristic $\mathrm{p},\left\{x_{1}, \ldots, x_{n}, \ldots\right\}$ a countably infinite set of indeterminates over $F$, $L=F\left(x_{1}, \ldots, x_{n}, \ldots\right)$ and $K=L^{p}\left(K\right.$ is the set of elements $\lambda^{p}$ where $\left.\lambda \in L\right)$. Let $y$ be an indeterminate over $L, V:=L[y]_{(y)}=L+y V$ and $R:=K+y V$. It is well known that $V$ is a rank-one discrete valuation domain, and $R$ is a one-dimensional SFT-PVD with maximal ideal $y V$ and associated valuation overring $V$. Since $L / K$ is purely inseparable with exponent $1, R\left[\left[x_{1}, \ldots, x_{n}\right]\right]$ is a Jaffard domain by Corollary 2 . But $R$ is not Noetherian since $L / K$ has infinite degree [5, Proposition 3.6].

Theorem 6 For any integral domain $R, \operatorname{dim}_{v} R[[x]] \geq 1+\operatorname{dim}_{v} R$.

Proof Let $T$ be an overring of $R$ and $A=T \times_{F} R[[x]]_{(x)}$ be the pullback of $T$ and $R[[x]]_{(x)}$ over $F$ where $F$ is the quotient field of $R$. By Fontana [8, Corollary 1.5-(6)], $A$ is an overring of $R[[x]]$, and so $\operatorname{dim}_{v} R[[x]] \geq$ $\operatorname{dim} A$. Since $R[[x]]_{(x)}$ is a rank-one valuation domain, $\operatorname{dim} A=\operatorname{dim} T+\operatorname{dim} R[[x]]_{(x)}=1+\operatorname{dim} T$ by Fontana [8, Proposition 2.1]. Hence, $\operatorname{dim}_{v} R[[x]] \geq 1+\operatorname{dim}_{v} R$.

Remark 7 All known examples of integral domains $R$ such that $R[[x]]$ is a Jaffard domain are $D$ and $\left.D\left[x_{1}\right]\right] \ldots$ $\left.\left[x_{m}\right]\right]$ (it is not necessary that $\left.\left[x_{i}\right]\right]=\left[\left[x_{i}\right]\right]$ for some $i$ ) where $\left\{x_{1}, \ldots, x_{m}\right\}$ is a finite set of indeterminates over $D$, and $D$ is:

(1) a finite-dimensional Noetherian domain, or

(2) a one-dimensional SFT-GPVD with associated Prüfer domain $T$ such that for each maximal ideal $M$ of $D$ with $M \supseteq I, K_{M}$ has finite exponent over $k_{0, M}$ and $\left[k_{0, M}: k_{M}\right]<\infty$ where $I=(D: T), N_{M}$ is the maximal ideal of $T$ such that $N_{M} \cap D=M, k_{M}:=D / M, K_{M}:=T / N_{M}$, and $k_{0, M}$ denotes the maximal separable extension of $k_{M}$ in $K_{M}$, or

(3) a one-dimensional APVD with SFT-integral closure such that $\left[K_{0}: K\right]<\infty$ and $L$ has finite exponent over $K_{0}$, where $K$ is the residue field of $D, L$ the residue field of the associated valuation domain of $D$ and $K_{0}$ the maximal separable extension of $K$ in $L$.

The most natural examples of Archimedean integral domains are arbitrary accp-domains (i.e., domains satisfying the ascending chain condition on principal ideals) and also Noetherian domains, one-dimensional domains and completely integrally closed domains. It is well known that if $R$ is an Archimedean domain, then so is the power series ring $R[[x]]$ and the polynomial ring $R[x]$. Therefore, all known examples of domains $R$ so that $R[[x]]$ is a Jaffard domain are Archimedean. Hence, it would be interesting to know if the following statement is true or not:

$$
R[[x]] \text { is a Jaffard domain } \Longrightarrow R \text { is Archimedean? }
$$

Also, all known examples of domains $R$ so that $R[[x]]$ is a Jaffard domain are accp-domains; indeed, it is well known that if $R$ is an accp-domain, then so is $R[[x]]$ [4, Lemma 2.1] and the polynomial ring $R[x]$. The remark is true for APVDs by Theorem 3 and it remains and suffices to show that a one-dimensional SFT-GPVD is 
an accp-domain. Let $R$ be a one-dimensional SFT-GPVD with associated Prüfer domain $T$. Note that $T$ is an SFT-ring [9, Proposition 1.7] and so is Noetherian. Since an element $r \in R$ is invertible in $R$ if and only if $\mathrm{r}$ is invertible in $T$, every increasing chain of principal ideals of $R$ stabilizes for some $n$. Thus, $R$ is an accp-domain. Hence, it would be interesting to know if the following statement is true or not:

$$
R[[x]] \text { is a Jaffard domain } \Longrightarrow R \text { is an accp-domain? }
$$

Finally, all known examples of domains $R$ so that $R[[x]]$ is a Jaffard domain are Jaffard domains. Hence, it would be interesting to know if the following statement is true or not:

$$
R[[x]] \text { is a Jaffard domain } \Longrightarrow R \text { is a Jaffard domain? }
$$

It is well known that if $R$ is an integral domain, then $\operatorname{dim} R[[x]] \geq 1+\operatorname{dim} R$. The last inequality may be equality (take $R$ to be Noetherian [7, Lemma 2.6]) and may be strict inequality (take $R=D[[y]]$ where $D$ is a rank-two SFT-valuation domain, then $\operatorname{dim} R[[x]]=2+\operatorname{dim} R$ [11, Theorem 14]). But all known examples of domains $R$ so that $\operatorname{dim}_{v} R[[x]]<\infty$ satisfy $\operatorname{dim}_{v} R[[x]]=1+\operatorname{dim}_{v} R$. Unfortunately, we are unable to find an example of domain $R$ such that $\infty>\operatorname{dim}_{v} R[[x]]>1+\operatorname{dim}_{v} R$.

Acknowledgments The author thanks the referees for their helpful comments and suggestions.

Open Access This article is distributed under the terms of the Creative Commons Attribution License which permits any use, distribution, and reproduction in any medium, provided the original author(s) and the source are credited.

\section{References}

1. Anderson, D.F.; Bouvier, A.; Dobbs, D.E.; Fontana, M.; Kabbaj, S.: On Jaffard domains. Exposit. Math. 6, 145-175 (1988)

2. Arnold, J.T.: Krull dimension in power series rings. Trans. Am. Math. Soc. 177, 299-304 (1973)

3. Badawi, A.; Houston, E.: Powerful ideals, strongly primary ideals, almost pseudo-valuation domains and conducive domains. Commun. Algebra 30(4), 1591-1606 (2002)

4. Buchsbaum, D.A.: Some remarks on factorization in power series rings. J. Math. Mech. 10(5), 749-753 (1961)

5. Cahen, P.J.: Construction B, I, D et anneaux localement ou résiduellement de Jaffard. Arch. Math. 54, 125-141 (1990)

6. Dobbs, D.E.; Fontana, M.: Locally pseudo-valuation domain. Ann. Mat. Pura Appl. 134, 147-168 (1983)

7. Fields, D.E.: Dimension theory in power series rings. Pac. J. Math. 35, 601-611 (1970)

8. Fontana, M.: Topologically defined classes of commutative rings. Annali Mat. Pura Appl. 123, 331-355 (1980)

9. Girolami, F.: Power series rings over globalized pseudo-valuation domains. J. Pure Appl. Algebra 50, 259-269 (1988)

10. Jaffard, P.: Théorie de la dimension dans l'anneau des polynômes. Mêm. Sci. Math. (Gauthier-Villars, Paris, 1960)

11. Kang, B.G.; Park, M.H.: Krull-dimension of mixed extensions. J. Pure Appl. Algebra 213(10), 1911-1915 (2009)

12. Khalifa, M.; Benhissi, A.: Krull dimension in power series ring over an almost pseudo-valuation domain. Commun. Algebra 38(8), 3014-3028 (2010)

13. Khalifa, M.; Benhissi, A.: SFT-stability and Krull dimension in power series ring over an almost pseudo-valuation domain. Annali Mat. Pura Appl. doi:10.1007/s10231-013-0360-8 (to appear)

14. Park, M.H.: Krull dimension of power series rings over a globalized pseudo-valuation domain. J. Pure Appl. Algebra 214(6), 862-866 (2010)

15. Park, M.H.: Krull dimension and generic fibres for mixed polynomial and power series integral domain. J. Algebra 358, 51-66 (2012) 